Bull. Austral. Math. Soc.

$16 \mathrm{~A} 99,16 \mathrm{~A} 21$

Vol. 41 (1990) [237-244]

\title{
ALMOST NILPOTENT GAMMA RINGS
}

\section{G.L. Booth and N.J. Groenewald}

In this paper we introduce the concept of almost nilpotence for $\Gamma$-rings, similar to the corresponding concept for rings, as defined by Van Leeuwen and Heyman. An almost nilpotent radical property $\mathcal{A}_{0}$ is introduced for $\Gamma$-rings, and shown to be supernilpotent. If $M$ is a $\Gamma$-ring with left and right operator rings $L$ and $R$ respectively, then $\mathcal{A}(L)^{+}=\mathcal{A}_{0}(M)=\mathcal{A}(R)^{*}$, where $\mathcal{A}(\mathrm{l})$ denotes the almost nilpotent radical of a ring. If $M$ is a $\Gamma$-ring and $m, n$ are positive integers, then $\mathcal{A}_{0}\left(M_{m, n}\right)$ is the almost nilpotent radical of the $\Gamma_{n, m}$-ring $M_{m, n}$.

\section{BASIC CONCEPTS}

Let $M$ and $\Gamma$ be additive abelian groups. If we have a map from $M \times \Gamma \times M$ to $M$ such that for all $x, y, z \in M, \gamma, \mu \in \Gamma$

(i) $x \gamma(y \mu z)=(x \gamma y) \mu z$;

(ii) $x \gamma(y+z)=x \gamma y+x \gamma z ; x(\gamma+\mu) y=x \gamma y+x \mu y$; $(x+y) \gamma z=x \gamma z+y \gamma z$

then $M$ is called a $\Gamma$-ring. If $U \subseteq M, V \subseteq M$ and $\varphi \subseteq \Gamma$ then we define:

$$
U \varphi V=\{u \gamma v: u \in U, \gamma \in \varphi, v \in V\}
$$

If $A$ is a subgroup of $M$, and $A \Gamma M \subseteq A, M \Gamma A \subseteq A$, then $A$ is an ideal of $M$, denoted by $A \unlhd M$. Similar notation will be used for ideals of rings. If $Q \unlhd M$, then $Q$ is called a semiprime ideal of $M$ if $A \unlhd M, A \Gamma A \subseteq Q \Rightarrow A \subseteq Q$. The next result is proved along the same lines as the corresponding result for rings:

Proposition 1.1. Let $M$ be a $\Gamma$-ring and let $Q \unlhd M$. Then the following are equivalent:

(a) $Q$ is a semiprime ideal of $M$;

(b) $\forall x \in M, x \Gamma M \Gamma x \subseteq Q \Longrightarrow x \in Q$.

If $A \unlhd M$, the factor $\Gamma$-ring $M / A$ is defined in the natural way.

Let $M, M^{\prime}$ be $\Gamma$-rings and let $f: M \rightarrow M^{\prime}$ be a mapping. If for all $x, y \in M$, $\gamma \in \Gamma, f(x+y)=f(x)+f(y)$ and $f(x \gamma y)=f(x) \gamma f(y)$, then $f$ is called a $\Gamma$-ring

Received 11 April 1989

Copyright Clearance Centre, Inc. Serial-fee code: 0004-9729/90 \$A2.00+0.00. 
homomorphism. If, in addition, $f$ is bijective, then $f$ is called a $\Gamma$-ring isomorphism. We will denote this by $M \cong M^{\prime}$. Similar notation will be used for rings.

If $A \unlhd M$, and $0 \neq I \unlhd M$ implies $I \cap A \neq 0$, then $A$ is called an essential ideal of $M$, denoted $A$ by $\triangleleft \cdot M$.

Let $x \in M, \gamma \in \Gamma$. Define $[x, \gamma]: M \rightarrow M$ by $[x, \gamma] y=x \gamma y$ for all $y \in M$. The subring $L$ of $\operatorname{End}(M)$ consisting of all sums $\sum_{i}\left[x_{i}, \gamma_{i}\right], x_{i} \in M, \gamma_{i} \in \Gamma$, is called the left operator ring of $M$. A right operator ring $R$ of $M$ is defined similarly, and consists of all sums of the form $\sum_{i}\left[\gamma_{i}, x_{i}\right], \gamma_{i} \in \Gamma, x_{i} \in M$.

$$
\begin{aligned}
\text { If } A \subseteq L, A^{*} & =\{x \in M \mid \forall \gamma \in \Gamma ;[x, \gamma] \in A\} . \\
\text { If } B \subseteq R, B^{*} & =\{x \in M \mid \forall \gamma \in \Gamma ;[\gamma, x] \in B\} . \\
\text { If } C \subseteq L, C^{+^{\prime}} & =\{\ell \in L \mid \ell M \subseteq C\} \text { and } \\
C^{*^{\prime}} & =\{r \in R \mid M r \subseteq C\} .
\end{aligned}
$$

It is easily seen that all of these mappings take ideals to ideals, and preserve intersections.

If $A \subseteq M$, then $[\Gamma, A]=\left\{\sum\left[\gamma_{i}, x_{i}\right] \mid \gamma_{i} \in \Gamma \& x_{i} \in M\right\}$. It is easily shown that, if $A \unlhd M$, then $[\Gamma, A] \unlhd R$ and that $[\Gamma, A] \subseteq A^{*^{\prime}}$. If $B \subseteq R$, then $M B=\left\{\sum x_{i} b_{i} \mid\right.$ $\left.x_{i} \in M, b_{i} \in B\right\}$. If $B \unlhd R$, then $M B \unlhd M$, and $M B \subseteq B^{*}$.

All classes of rings considered will be for some fixed $\Gamma$, and abstract, that is closed under isomorphisms. A radical property $\mathcal{R}$ for $\Gamma$-rings is defined as for the corresponding concept for rings (see $[7, \mathrm{p} .3]$ ). If $\mathcal{U}$ is a class of rings, the lower radical determined by $\mathcal{U}$ is constructed as for rings [7, p.13]). A class $\mathcal{C}$ of $\Gamma$-rings is called weakly special, if:

(a) $\mathcal{C}$ consists of semiprime $\Gamma$-rings;

(b) if $m \in \mathcal{C}$ and $A \unlhd M$, then $A \in \mathcal{C}$;

(c) if $M$ is a $\Gamma$-ring and $A \unlhd \cdot M$, then $A \in \mathcal{C}$ implies $M \in \mathcal{C}$.

A weakly special class of rings is defined in the same way.

Proposition 1.2. ([5, Theorem 2.5]). Let $\mathcal{C}$ be a weakly special class of rings. Let $\overline{\mathcal{C}}$ be the class of all $\Gamma$-rings $M$ such that the right operator ring $\mathcal{R}$ of $M$ is in $\mathcal{C}$, and $M \Gamma x=0$ implies $x=0$, for all $x \in M$. Then $\overline{\mathcal{C}}$ is a weakly special class of gamma rings.

If $\mathcal{C}$ is a weakly special class of $\Gamma$-rings, the upper radical $\mathcal{R}$ determined by $\mathcal{C}$ is

$$
\mathcal{R}=\mathcal{U C}=\{M \mid M \text { has no nonzero homomorphic image in } \mathcal{C}\} .
$$

A radical property $\mathcal{R}$ for $\Gamma$-rings is called supernilpotent if $\mathcal{R}$ includes all $\Gamma$-rings $M$ such that $M \Gamma M=0$, and if $M \in \mathcal{R}$, then $A \unlhd M$ implies $A \in \mathcal{R}$. As for rings we have: 
Proposition 1.3. A radical property $\mathcal{R}$ for $\Gamma$-rings is supernilpotent if and only if $\mathcal{R}=\mathcal{U C}$ for some weakly special class $\mathcal{C}$ of $\Gamma$-rings $M, \mathcal{R}(M)=\cap\{Q \unlhd M \mid$ $M / Q \in \mathcal{C}\}$.

\section{AlMOST NILPOTENT RINGS}

Almost nilpotent rings were introduced by Van Leeuwen and Heyman [12]. A ring $R$ is called almost nilpotent (anp) if $(\forall 0 \neq A \unlhd R)(\exists n \in N)\left(R^{n} \subset A\right)$. This definition, which first appeared in [8], is slightly stronger than the one given in [12]. Nevertheless, all the results concerning anp rings which are discussed in this paper remain valid for this definition. The lower radical property $\mathcal{A}$ determined by the class of all anp rings is supernilpotent, and coincides with the upper radical property determined by the class $\mathcal{C}$ of rings which contain no nonzero anp ideals (see [12, Theorems 2 and 4]). $\mathcal{A}$ is called the almost nilpotent radical property in the variety of rings.

A $\Gamma$-ring $M$ is called anp if $(\forall 0 \neq A \unlhd M)(\exists n \in N)(M \Gamma)^{n} M=M \Gamma M \Gamma \ldots \Gamma M \subset$ $A$. Let $\mathcal{A}_{0}$ be the lower radical property determined by the class of anp $\Gamma$-rings. Since the class of anp $\Gamma$-rings contains all $\Gamma$-rings $M$ such that $M \Gamma M=0$, the construction of $\mathcal{A}_{0}$ terminates at the second step ([10, Lemma 2.4]), that is $\mathcal{A}_{0}$ is the set of all $M$ such that every nonzero homomorphic image of $M$ has a non-zero ideal which is anp.

$\mathcal{A}_{0}$ is called almost nilpotent radical property for $\Gamma$-rings. Let $\mathcal{C}_{0}$ denote the class of all $\Gamma$-rings which contain no nonzero anp ideals. Throughout this section, let $M$ denote a $\Gamma$-ring with left and right operator rings $L$ and $R$, respectively.

Proposition 2.1. Suppose that $A$ is an anp ideal of $M$. Then $[\Gamma, A]$ is an anp ideal of $R$ :

Proof: Let $0 \neq B \unlhd[\Gamma, A]$. We claim that $M B \unlhd A$. For if $x \in M, b \in B$, then $b=\sum_{i}\left[\alpha_{i}, a_{i}\right]$ for some $\alpha_{i} \in \Gamma, a_{i} \in A$. Hence $x b=\sum_{i} x \alpha_{i} a_{i} \in A$ since $A \unlhd M$. Thus $M B \subseteq A$. Since $B \neq 0, M B \neq 0$. Now suppose $x \in M, b \in B, a \in A$ and $\gamma \in \Gamma$. Then $a \gamma(x b)=(a \gamma x) b \in M B$, and $(x b) \gamma a=x(b[\gamma, a]) \in M B$ since $B \unlhd[\Gamma, A]$. Thus $0 \neq M B \unlhd A$. Since $A$ is an anp ideal of $M,(A \Gamma)^{n} A \subset M B$ for some $n \in \mathbb{N}$. Hence, $A[\Gamma, A]^{n} \subset M B$, whence $[\Gamma, A]^{n+1} \subseteq[\Gamma, M B]=R B$. It follows that $[\Gamma, A]^{n+2} \subseteq[\Gamma, A] R B \subseteq[\Gamma, A] B \subseteq B$ since $B \unlhd[\Gamma, A]$.

Since $(A \Gamma)^{n} A \subset M B$, there exist $x \in M, b \in B$ such that $x b \notin(A \Gamma)^{n} A$. Suppose that $b \in[\Gamma, A]^{n+2}$. Then there exist $a_{i j} \in A, \gamma_{i j} \in \Gamma, 1 \leqslant i \leqslant n+2,1 \leqslant j \leqslant m$ such that

$$
b=\sum_{j=1}^{m}\left[\gamma_{1 j}, a_{1 j}\right] \ldots\left[\gamma_{n+2 j}, a_{n+2 j}\right]
$$


Hence

$$
x b=\sum_{j=1}^{m}\left(x \gamma_{1 j} a_{1 j}\right) \gamma_{2 j} a_{2 j} \ldots \gamma_{n+2 j} a_{n+2 j} \in(A \Gamma)^{n} A,
$$

which contradicts our choice of $x$ and $b$. Hence $b \notin[\Gamma, A]^{n+2}$, so $[\Gamma, A]^{n+2} \subset B$, as required.

Proposition 2.2. Suppose that $M$ has the property that $(\forall x \in M)(M \Gamma x=0 \Longrightarrow x=0)$. Let $A$ be an anp ideal of $R$. Then $A^{*}$ is an anp ideal of $M$.

Proof: It is easily verified that $\left[\Gamma, A^{*}\right] \subseteq A$. Since $\left[\Gamma, A^{*}\right] \triangleleft R,\left[\Gamma, A^{*}\right] \triangleleft A$. But the class anp rings is hereditary (see [12]), the Lemma before Theorem 2). Hence, $\left[\Gamma, A^{*}\right]$ is an anp ideal of $R$. Suppose $0 \neq B \triangleleft A^{*}$. If $[\Gamma, B]=0$, then $M \Gamma B=0$, whence $B=0$, contradicting our choice of $B$. Thus $0 \neq[\Gamma, B] \triangleleft\left[\Gamma, A^{*}\right]$. Hence there exists $n \in \mathrm{N}$ such that $\left[\Gamma, A^{*}\right]^{n} \subset[\Gamma, B]$. It follows that $\left(A^{*} \Gamma\right)^{n} A^{*} \subseteq A^{*} \Gamma B \subseteq B$ since $B \triangleleft A^{*}$. Since $\left[\Gamma, A^{*}\right]^{n} \subset[\Gamma, B]$, there exist $\gamma \in \Gamma, b \in B$ such that $[\gamma, b] \notin\left[\Gamma, A^{*}\right]^{n}$. Then $b \notin\left(A^{*} \Gamma\right)^{n} A^{*}$, otherwise $[\gamma, b] \in\left[\gamma, A^{*}\right]^{n+1} \subseteq\left[\Gamma, A^{*}\right]^{n}$, contradicting our choice of $\gamma$ and $b$. Hence $\left(A^{*} \Gamma\right)^{n} A^{*} \subset B$, as required.

THEOREM 2.3. The following are equivalent:

(a) $M$ contains no nonzero anp ideals;

(b) $R$ contains no nonzero anp ideals, and $(\forall x \in M)(M \Gamma x=0 \Longrightarrow x=0)$;

(c) $L$ contains no nonzero anp ideals, and $(\forall x \in M)(x \Gamma M=0 \Longrightarrow x=0)$.

Proof:

(a) $\Longrightarrow$ (b): Suppose $M$ contains no nonzero anp ideals. Clearly, $M$ is a semiprime $\Gamma$-ring. Suppose $x \in M$ and $M \Gamma x=0$. Then $x \Gamma M \Gamma x=0$, whence $x=0$. Suppose that $A$ is an anp ideal of $R$. Then $A^{*}$ is an anp ideal of $M$, by Proposition 2.2. Hence, $A^{*}=0$. Now $A \subseteq\left(A^{*}\right)^{*^{\prime}}=0^{*^{\prime}}=\{r \in R: M r=0\}=0$, as required.

(b) $\Longrightarrow$ (a): Suppose that $R$ contains no nonzero anp ideals, and that $(\forall x \in M)(M \Gamma x=0) \Rightarrow x=0$. Let $A$ be an anp ideal of $M$. Then $[\Gamma, A]$ is an anp ideal of $R$, by Proposition 2.1. Hence $[\Gamma, A]=0$, whence $M \Gamma A=0$. It follows that $A=0$.

(a) $\Longrightarrow$ (c) follows by symmetry.

Let $\mathcal{C}_{0}$ denote the class of all $\Gamma$-rings without anp ideals. As an immediate consequence of Proposition 1.2 and Theorem 3.3, we have

COROLlary 2.4. $C_{0}$ is a weakly special class of $\Gamma$-rings.

Proposition 2.5. $\mathcal{A}_{0}=\mathcal{U C}_{0}$.

The proof is identical to that of the corresponding result for rings ([12, Theorem 4]). 
From Corollary 2.4 and Proposition 2.5, we have

THEOREM 2.6. $\mathcal{A}_{0}$ is a supernilpotent radical property in the variety of $\Gamma$-ring.

It follows that $\mathcal{A}_{0}(M)=\cap\left\{Q \unlhd M \mid M / Q \in \mathcal{C}_{0}\right\}$.

Lemma 2.7. ([3, Corollary 2.2]). Let $A \triangleleft M$, and let $R^{\prime}$ denote the right operator ring of the factor $\mathrm{\Gamma}$-ring $M / A$. Then

$$
R^{\prime} \cong R / A^{*^{\prime}} \text {. }
$$

Leмma 2.8. The mapping $Q \rightarrow Q^{*}$ defines a one-to-one correspondence between ideals $Q$ of $R$ such that $Q / R \in \mathcal{C}$ and ideals $S$ of $M$ such that $M / S \in \mathcal{C}_{0}$.

Proof: Let $Q \unlhd R$ be such that $R / Q \in \mathcal{C}$. Then $Q$ is a semiprime ideal of $R$. Hence $Q=\left(Q^{*}\right)^{*^{\prime}}$ (see [9, Theorem 1]). The right operator ring $R^{\prime}$ of the factor $\Gamma$-ring $M / Q^{*}$ is isomorphic to $R /\left(Q^{*}\right)^{*^{\prime}}=R / Q$, by Lemma 2.7. Suppose $x \in M$ is such that $\left(M / Q^{*}\right) \Gamma\left(x+Q^{*}\right)=0$. It follows that $M \Gamma x \subseteq Q^{*}$. Since $Q$ is a semiprime ideal of $R$, $Q^{*}$ is a semiprime ideal of $M([\boldsymbol{\theta}$, Lemma 1$])$. Now $x \Gamma M \Gamma x \subseteq Q^{*}$, whence $x \in Q^{*}$, that is $x+Q^{*}=0$. By Theorem 2.3, $M / Q^{*}$ contains no nonzero anp ideals, whence $M / Q \in \mathcal{C}_{0}$.

Conversely, suppose $S \triangleleft M$ is such that $M / S \in \mathcal{C}_{0}$. Since $S$ is a semiprime ideal of $M, S=\left(S^{*}\right)^{*}$. Since the right operator ring of the factor $\Gamma$-ring $M / S$ is isomorphic to $R / S^{*^{\prime}}$, by Lemma 2.7 , it follows from Theorem 2.3 that $R / Q^{*^{\prime}} \in \mathcal{C}$, as required. $]$

THEOREM 2.9. $\mathcal{A}(R)^{*}=\mathcal{A}_{0}(M)=\mathcal{A}_{0}(L)^{+}$.

Proof: $\mathcal{A}(R)=\mathcal{A}_{0}(M)$ follows easily from Lemma 2.8. It follows by symmetry that $\mathcal{A}(L)^{+}=\mathcal{A}_{0}(M)$.

\section{MATRIX T-RINGS}

Let $M$ be a $\Gamma$-ring, and let $m, n$ be positive integers. Denote by $M_{m, n}$ and $\Gamma_{n, m}$, the sets of $m \times n$ matrices with entries from $R$, and $n \times m$ matrices with entries from $\Gamma$, respectively. Then $M_{m, n}$ is a $\Gamma_{n, m}$-ring with the matrix addition, and multiplication defined by

where

$$
\left(a_{i j}\right)\left(\gamma_{i j}\right)\left(b_{i j}\right)=\left(c_{i j}\right), c_{i j}=\sum_{p} \sum_{q} a_{i p} \gamma_{p q} b_{q j} .
$$

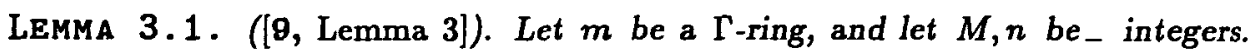
Denote by $R^{\prime}$ the right operator ring of the matrix $\Gamma_{n, m}$-ring $M_{m, n}$. Then $R^{\prime} \cong R_{n}$, the ring of $n \times n$ matrices with entries from $R$. Moreover, if $A \subseteq M$, then $\left(A_{n}\right)^{*}=\left(A^{*}\right)_{m, n}$.

LemMA 3.2. ([8, Theorem 3]). Let $R$ be a ring, and let $n$ be a positive integer. Then

$$
\mathcal{A}\left(R_{n}\right)=(\mathcal{A}(R))_{n}
$$


Theorem 3.3. Let $M$ be a $\Gamma$-ring, and let $m, n$ be positive integers. Then

$$
\mathcal{A}_{0}\left(M_{m, n}\right)=\left(\mathcal{A}_{0}(M)\right)_{m, n}
$$

Proof:

$$
\begin{aligned}
\mathcal{A}_{0}\left(M_{m, n}\right) & =\left(\mathcal{A}\left(R_{n}\right)\right)^{*} & & \text { (by Theorem 2.9) } \\
& =\left((\mathcal{A}(R))_{n}\right)^{*} & & \text { (by Lemma 3.2) } \\
& =\left(\mathcal{A}(R)^{*}\right)_{m, n} & & \text { (by Lemma 3.1) } \\
& =\left(\mathcal{A}_{0}(M)\right)_{m, n} & & \text { (by Theorem 2.9). }
\end{aligned}
$$

Every ring $R$ is a $\Gamma$-ring with $\Gamma=R$ and the usual addition and multiplication operations on $R$. Let $R^{\prime}$ denote the right operator ring of $R$ considered as a $\Gamma$-ring with $\Gamma=R$. Define $f: R^{2} \rightarrow R^{\prime}$ by

$$
f\left(\sum_{i} x_{i} y_{i}\right)=\sum_{i}\left[x_{i}, y_{i}\right]
$$

It is easily shown that $f$ is well-defined, and maps the ring $R^{2}$ homomorphically onto $R^{\prime}$. Moreover, the kernel $K$ of $f$ is equal to $R^{2} \cap r(R)$, where $r(R)$ denotes the right annihilator of $R$. Hence,

$$
R^{\prime} \cong \frac{R^{2}}{K}=\frac{R^{2}}{R^{2} \cap r(R)}
$$

LEMMA 3.4. Let $\mathcal{R}$ be any supernilpotent radical property. Then

$$
\mathcal{R}\left(R^{\prime}\right)=\frac{R^{2} \cap \mathcal{R}(R)}{R^{2} \cap r(R)}=\frac{R^{2} \cap \mathcal{R}(R)}{K}
$$

Proof: Note firstly that $K$ is a zero ring. Since $\mathcal{R}$ is a supernilpotent radical property, $K \in \mathcal{R}$ and hence $K \subseteq \mathcal{R}\left(R^{2}\right)=R^{2} \cap \mathcal{R}(R)$. Let $\mathcal{R}\left(R^{\prime}\right)=A / K$ where $K \subseteq A \unlhd R^{2}$. Let $f$ be the natural homomorphism of $R^{2}$ onto the factor ring $R^{2} / K$. Then $f\left(\mathcal{R}\left(R^{2}\right)\right) \subseteq \mathcal{R}\left(R^{\prime}\right)$, whence $R^{2} \cap \mathcal{R}(R) \subseteq A$. But $K \in \mathcal{R}$ and $A / K=\mathcal{R}\left(R^{\prime}\right) \in$ $\mathcal{R}$, whence by the extension property, $A \in \mathcal{R}$. It follows that $A \subseteq \mathcal{R}\left(R^{2}\right)$. Hence $A=R^{2} \cap \mathcal{R}(R)$, and the result follows.

Theorem 3.5. Let $R$ be a ring. Then $\mathcal{A}(R)=\mathcal{A}_{0}(R)$, where $\mathcal{A}$ and $\mathcal{A}_{0}$ denote, respectively, the anp radical of the ring $R$ and the anp radical of $R$ considered as a $\Gamma$-ring with $\Gamma=R$.

Proof: Let $R^{\prime}=R^{2} / K$ be the right operator ring of $R$ considered as a $\Gamma$ ring with $\Gamma=R$, where $K=R^{2} \cap r(R)$. Then by Theorem $2.9, \mathcal{A}_{0}(R)=\mathcal{A}\left(R^{\prime}\right)^{*}$. 
We will show that $\mathcal{A}\left(R^{\prime}\right)^{*}=\mathcal{A}(R)$. Let $x \in \mathcal{A}(R)$. Then, for all $r \in R, r x \in$ $R^{2} \cap \mathcal{A}(R)$, whence $r x+K \in R^{2} \cap \mathcal{A}(R) / K=\mathcal{A}\left(R^{\prime}\right)$, by Lemma 3.4. Hence $x \in \mathcal{A}\left(R^{\prime}\right)^{*}$. Conversely, suppose that $y \in \mathcal{A}\left(R^{\prime}\right)^{*}$. Then $r y+K \in \mathcal{A}\left(R^{\prime}\right)=R^{2} \cap \mathcal{A}(R) / K$, for all $r \in R$. It follows that $R y \subseteq \mathcal{A}(R)$, whence $y R y \subseteq \mathcal{A}(R)$. Since $\mathcal{A}(R)$ is a semiprime ideal of $R$, this implies that $y \in \mathcal{A}(R)$. Thus $\mathcal{A}\left(R^{\prime}\right)^{*}=\mathcal{A}(R)$.

Let $R$ be a ring, and let $m, n$ be positive integers. Let $R_{m, n}$ denote the set of $m \times n$ matrices with entries from $R$. Then clearly $R_{m, n}$ is an $R_{n, m}$-ring with the usual operations of matrix addition and multiplication.

Theorem 3.6. Let $R$ be a ring, and let $m, n$ be positive integers. Then

$$
\mathcal{A}_{0}\left(R_{m, n}\right)=(\mathcal{A}(R))_{m, n}
$$

where $\mathcal{A}_{0}\left(R_{m, n}\right)$ denotes the anp radical of the $R_{n, m}$-ring $R_{m, n}$.

The proof is an easy consequence of Theorems 3.3 and 3.5, and will be omitted.

\section{Position OF THE ANP RADICAL}

The prime, Jacobson and antisimple radicals $\beta, \mathcal{J}$ and $\beta_{\phi}$ were defined for $\Gamma$-rings in [6] and [4], respectively. We will use the same notation for the corresponding radical properties of rings.

Proposition 4.1. Let $\mathcal{R}$ denote the prime, Jacobson or antisimple radical property for a ring, and let $\mathcal{R}_{0}$ denote the corresponding radical property for a $\Gamma$-ring. Let $M$ be an arbitrary $\Gamma$-ring with right operator ring $R$. Then $\mathcal{R}(R)^{*}=\mathcal{R}_{0}(M)$.

For the proof, we refer to [6, Theorems 4.1 and 8.2], and [4, Corollary 2.10].

It is known [12, Theorem 5] that for a ring $R, \beta(R) \subseteq \mathcal{A}(R) \subseteq \beta_{\phi}(R)$. As an easy consequence of this result, Theorem 2.9 and Proposition 4.1, we have

Proposition 4.2. Let $M$ be a $\Gamma$-ring. Then $\beta(M) \subseteq \mathcal{A}_{0}(M) \subseteq \beta_{\phi}(M)$.

Analogues of Theorem 3.5 are known for the prime and Jacobson radicals ([1, Theorem 4.7] and [6, Theorem 10.1] respectively). In [4, Theorem 2.4 and Corollary 2.10] respectively, it is shown that $\beta_{\phi}$ is a special radical property in the variety of $\Gamma$-rings, and that for a $\Gamma$-ring $M$ with right operator ring $R, \beta_{\phi}(R)^{*}=\beta_{\phi}(M)$. Using the techniques of proof employed in Lemma 3.4 and Theorem 3.5, it is easy to prove the following result

Lemma 4.3. Let $R$ be a ring and let $\beta_{\phi}(R)$ and $\beta_{\phi}^{\prime}(R)$ denote, respectively, the antisimple radicals of the ring $R$ and of $R$ considered as a $\Gamma$-ring with $\Gamma=R$. Then $\beta_{\phi}(R)=\beta_{\phi}^{\prime}(R)$.

For rings it is known (see [12, Theorem 5] and [11, Example 2]) that the inclusion $\beta \subset \mathcal{A} \subset \beta_{\phi}$ is strict and that $\mathcal{A}$ and $\mathcal{J}$ are independent. In view of Theorem 3.5, 
its analogues for the prime and Jacobson radicals and Lemma 4.3, we have for $\Gamma$-rings that the inclusion $\beta \subset \mathcal{A}_{0} \subset \beta_{\phi}$ is strict and that the radical properties $\mathcal{A}_{0}$ and $\mathcal{J}$ are independent.

\section{REFERENCES}

[1] G.L. Booth, A contribution to the radical theory of gamma rings (Ph.D. thesis, University of Stellenbosch, 1986).

[2] G.L. Booth, 'Characterizing the nil radical of a gamma ring', Quasestiones Math 9 (1986), 55-67.

[3] G.L. Booth, 'Operstor rings of a gamma ring', Math. Japon. 31 (1986), 175-184.

[4] G.L. Booth On antisimple gamma rings, Quaestiones Math. 11 (1988), 7-15.

[5] G.L. Booth, 'Supernilpotent radicals of gamma rings', Acta. Math. Hungar. (to appear).

[6] W.E. Coppage and J. Luh, 'Radicals of gamma rings', J. Math. Soc. Japan 23 (1971), 40-52.

[7] N. Divinsky, Rings and Radicals (Univ. of Toronto Press, Toronto, 1965).

[8] G.A.P. Heyman, T.L. Jenkins and H.J. Le Roux, 'Variations on almost nilpotent rings, their radicals and partitions', Acta. Math. Hungar. 39 (1982), 11-15.

[9] S. Kyuno, 'Prime ideals in gamma rings', Pacific J. Math. 88 (1982), 375-379.

[10] H.J. Le Roux, Lower radicals of $\Gamma$-rings, (Preprint).

[11] G. Tzintzis, 'An upper radical property and an answer to a problem of L.C.A. van Leeuwen and G.A.P. Heyman', Acta. Math. Hungar 38 (1982), 381-385.

[12] L.C.A. Van Leeuwen and G.A.P. Heyman, 'A class of almost nilpotent rings', Tomus, Acta. Math. Hungar. 26 (1975), 259-262.

Department of Mathematics

University of Zululand

Private Bag X1001

Kwadlangezwa 3886

South Africa
Department of Mathematics

University of Port Elizabeth

P.O. Box 1600

Port Elizabeth 6000

South Africa 